\title{
A TURN BY TURN PHOTON BEAM PROFILE OBSERVATION BY A LASER BEAM SATURATED PHOTODIODE
}

\author{
Ian C. Hsu, C.-H. Lee, and G.-T. Cheng \\ Department of Nuclear Science, National Tsing Hua University and SRRC, TAIWAN
}

\begin{abstract}
A fast photodiode was demonstrated to be able to extract the turn by turn photon beam profile information of electron storage rings if the photodiode was operated at the saturated region. When applied this method to a 1.3 $\mathrm{GeV}$ synchrotron light source with $200 \mathrm{~mA}$ beam current, the synchrotron radiation intensity is not intense enough to saturate the photodiode. To apply this method to the type of light source as previously mentioned, a flat-top laser beam was constructed to saturate the photodiode. The theory and the preliminary experimental results are presented and discussed.
\end{abstract}

\section{INTRODUCTION}

In many accelerator physics experiments, e.g., the coherent damping time measurement and the dynamic aperture study experiments, we like to know the turn by turn variations of the beam positions. Usually, it is accomplished by the button type or the strip line type electrode beam position monitors (BPM). However, the measurements done by these types of BPM only gave us the information of the position of the beam centroid. If the beam centroid motion combined with the decoherence mechanism, the BPM would not be able to distinguish them. This means that by using the BPM, we can not distinguish the coherence damping or the decoherence. In order to distinguish them, we need to monitor the beam profile simultaneously. For lepton machines, the synchrotron radiation provides a very useful beam profile information. However, to perform a turn by turn beam profile monitor, we need a very fast detecting system. For the speed requirement we need, the commercial photodiode array and the following up electronic system is not available neither a cost reasonable approach. A method has been proposed, to monitor the beam profile by using a fast single photo diode.[1] The idea has been examined by using a modulated He-Ne laser to simulate the synchrotron radiation light. The results agree with computer simulation results. When applied this method to a $1.3 \mathrm{GeV}$ synchrotron light source with $200 \mathrm{~mA}$ beam current, the synchrotron radiation intensity is not intense enough to saturate the photodiode. To apply this method to the type of light source as previously mentioned, a flattop laser beam was constructed to saturate the photodiode. The theory and the preliminary experimental results are presented and discussed.

\section{THEORY}

Most P-N silicon photodiodes are linear (better than $1 \%$ ) over a wide range of magnitude of the incident power. In linear region, the total photocurrent is independent of the incident photon beam size as long as the total power is the same. At high photon intensity, however, nonlinearity is introduced due to the device saturation. Total photocurrent in nonlinearity region now is not only dependent on the incident total photon power but also dependent upon the photon beam size. This means that in the nonlinearity region, we can get the photon beam profile information by means of measuring the total photocurrent. More detailed discussions and computer simulations can be found in a previous paper.[1] However, we were not able to use the system to measure the beam size variation of the type of light source as previously mentioned. It was due to that the intensity of the synchrotron light is 10 to 100 times lower than that of the laser which we used in the experiments. To solve this problem, we can build a flat-top beam as a saturating beam. With the saturating beam, we can force the photodiode operated in the non-linear region. In this way, for either the case of a weak synchrotron light or the case of a larger beam size variation range i.e. larger photon intensity variation range, the detector system can detect the beam size variations. That means that we enlarge the dynamic range of the system. If the saturating beam is a gaussian beam (TEMoo mode), then, if the detected beam has a position variation but not the beam size variation, the detector system will still give us changing output signal which will cause confuse. That is reason why we need a flat-top beam as a saturating beam.

\section{CONSTRUCTION OF FLAT TOP LASER BEAMS}

Absorption method was used for this purpose. We photograph the expended gaussian beam. If we can manipulate the Gamma value of the film to be 1, when the expended gaussian beam passed the developed film, a flat-top beam can be obtained.

Figure 1 shown a theoretical Hurter-Driffield curve[2]. The curve describes the general relation between the Exposure (E) of the film to the light source and the photographic Density (D) after the film was developed. There is a linear region in the curve. The 


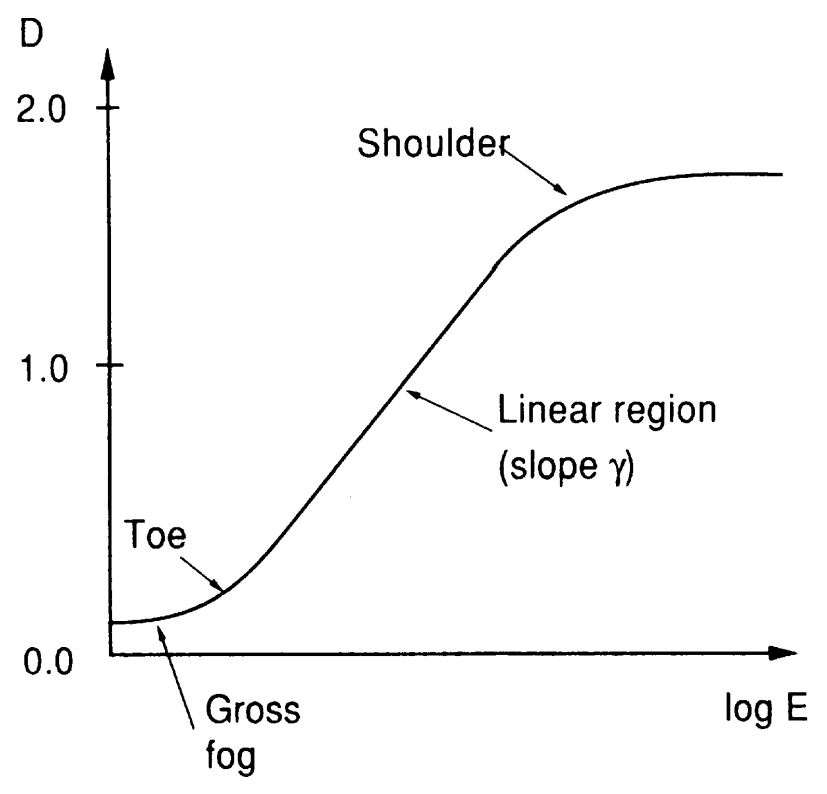

Fig. 1 The theoretical Hurter-Driffield curve

slope of the linear part is define to be the Gamma value of the film. It was shown[3] that if the exposure of the whole Gaussian beam as well as the development process was controlled such that the whole exposure area was remained inside the linear region and with the slop equals to 1 , we can produce a flat-top beam by sending a gaussian beam with the same beam size through the film. Figure 2 is the Hurter-Driffield curve of one of the film which we produced. The horizontal axis is the photon flux $(\mathrm{P})$ times the exposure time $(\mathrm{t})$ which is proportional to the Exposure. The Gamma value equals to 1.072 . Figure 3 is the flat-top gaussian beam produced by the same film which the Fig. 2 was measured from.

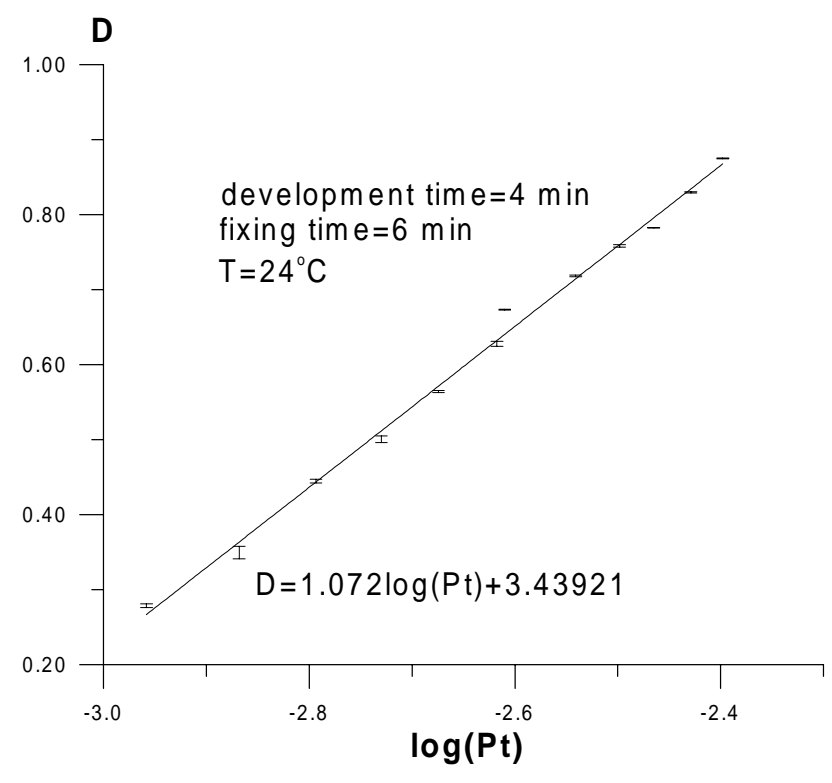

Fig. 2 The Hurter-Driffield curve of one of the film with Gamma value equals to 1.072

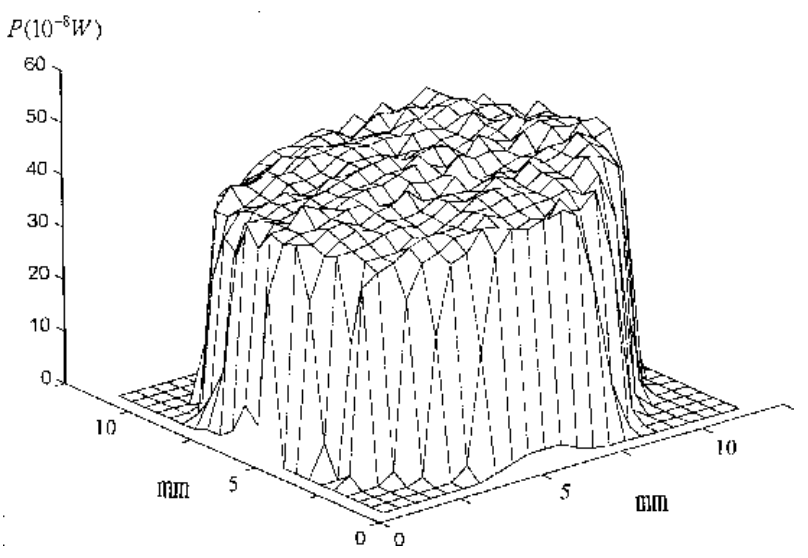

Fig. 3 The measurement results of the flat-top beam

\section{EXPERIMENTS}

The schematic drawing of the experimental setup was shown in Fig. 4.

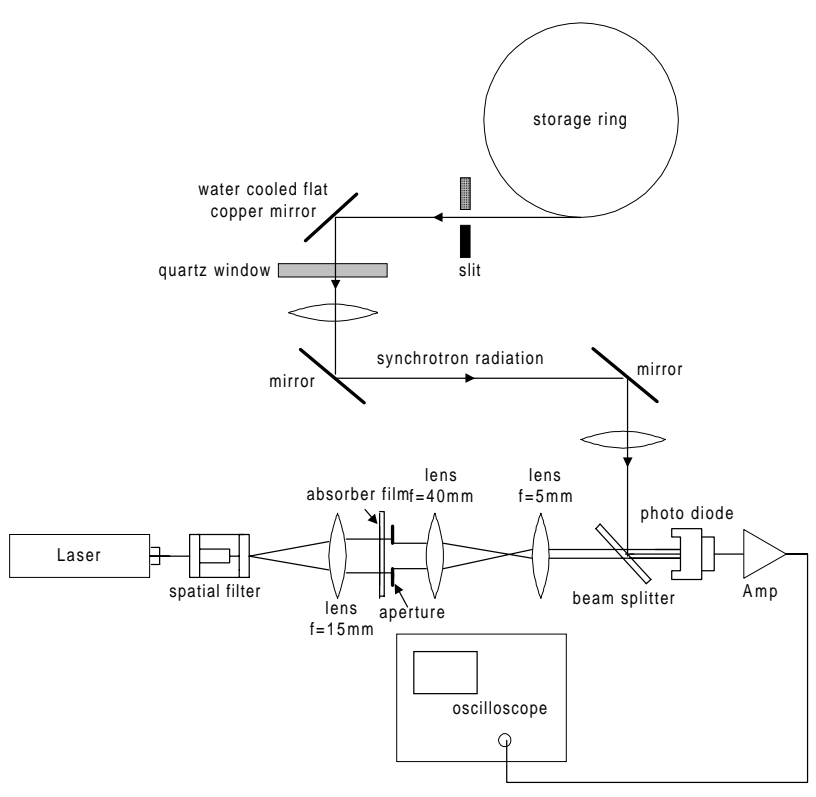

Fig.4 The schematic drawing of the experimental setup

To observe a predictable fast beam size varation, we fire one of the injection kickers. Due to the energy spread and the no-zero chromaticity value, the betatron oscillation of electrons inside the bunch will start to dechroherent, and increase the beam size. After one synchrotron oscillation period the bunch will recoherence again and the beam size shrank to the original one.[4] Then the process repeat itself again. The larger the value of the chromaticity the deeper the beam size variation will be.

Figures 5, 6 and 7 are the photodiode measurement results for different sextupole values. We see the beam size variation with a period of 30 microsecond, which is the synchrotron oscillation period of the storage ring under the experiment. 


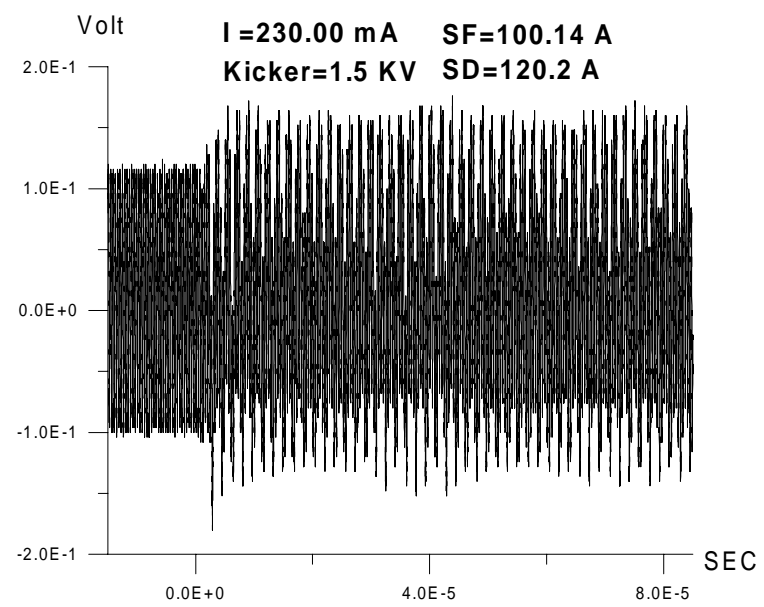

Fig. 5 Photodiode measurement results for smaller chromaticity value

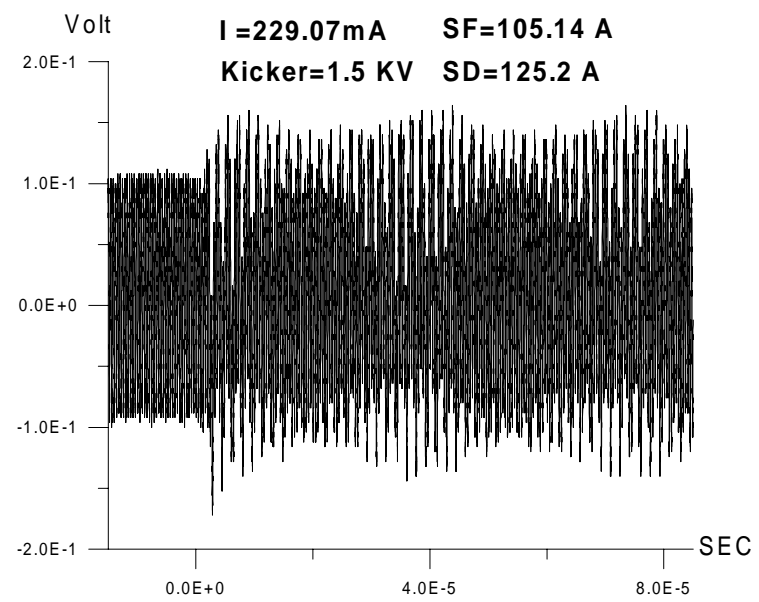

Fig. 6 Photodiode measurement results for medium chromaticity value

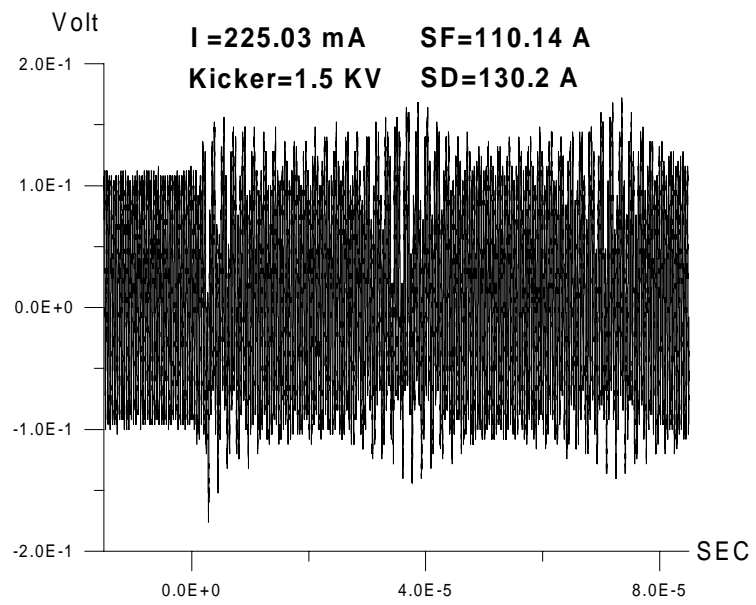

Fig. 7 Photodiode measurement results for larger chromaticity value

\section{DISCUSSION AND CONCLUSSION}

From the preliminary experiment results, it does show that the fast photodiode can provide turn by turn beam size information, if the photodiode operated in the nonlinear region. However, in this preliminary test, instead of using single bunch beam, we have used multibunch beam. The reason is that the efficiency of the production of flat-top beam is too low (12\%, in our case), therefore, it is not strong enough to saturate the photodiode in the single bunch beam (typical beam current is $20 \mathrm{~mA}$ ). The different method of producing flat-top beam is under study which should overcome this weak point.

\section{ACKNOWLEDGEMENTS}

We would like to thank Dr. K.-T. Hsu of SRRC, Taiwan, for his help in providing the BPM signal and the optics system of the synchrotron radiation monitor. This work was supported by the NSC of Taiwan, under contract NSC 85-2112-M-007-019.

\section{REFERENCES}

[1] Ian C. Hsu et al., in Proceeding of the 1995 Particle Accelerator Conference (Dallas, Texas, USA, 1995), pp. 2515-2517.

[2] J.W.Goodman,Introduction to Fourier Optics (McGraw-Hill,New York,1996),Chap.7,p. 176.

[3] Ian and C.-H. Lee, to be published.

[4] Ian C. Hsu, Particle Accelerators 34(12) (1990) 43 